\title{
Haploinsufficiency of the autism candidate gene Neurobeachin induces autism-like behaviors and affects cellular and molecular processes of synaptic plasticity in mice
}

\author{
Kim Nuytens ${ }^{\text {a,b, } 1}$, Ilse Gantois ${ }^{\text {c, } 1}$, Pieter Stijnen ${ }^{a}$, Emilia Iscru ${ }^{c}$, Annelies Laeremans ${ }^{\mathrm{d}}$, Lutgarde Serneels ${ }^{\text {e,f }}$, \\ Lien Van Eylen ${ }^{\text {b,g }}$, Stephen A. Liebhaber ${ }^{\mathrm{h}}$, Koen Devriendt ${ }^{\mathrm{b}, \mathrm{i}}$, Detlef Balschun ${ }^{\mathrm{c}}$, Lutgarde Arckens ${ }^{\mathrm{d}}$, \\ John W.M. Creemers ${ }^{a, b, *, 2}$, Rudi D'Hooge ${ }^{c, 2}$
}

\footnotetext{
a Laboratory for Biochemical Neuroendocrinology, Department of Human Genetics, KU Leuven, 3000 Leuven, Belgium

${ }^{\mathrm{b}}$ Leuven Autism Research Consortium (LAuRes), KU Leuven, 3000 Leuven, Belgium

c Laboratory of Biological Psychology, Department of Psychology, KU Leuven, 3000 Leuven, Belgium

d Laboratory of Neuroplasticity and Neuroproteomics, Department of Biology, KU Leuven, 3000 Leuven, Belgium

e Laboratory for the Research of Neurodegenerative Diseases, Department of Human Genetics, KU Leuven, 3000 Leuven, Belgium

${ }^{\mathrm{f}}$ Vlaams Instituut voor Biotechnologie (VIB), Belgium

' Parenting and special education research group, Child Psychiatry, UZ Leuven, 3000 Leuven, Belgium

${ }^{\mathrm{h}}$ Departments of Genetics and Medicine, University of Pennsylvania, Philadelphia, PA 19104, USA

${ }^{\mathrm{i}}$ Laboratory for Genetics of Human Development, Department of Human Genetics, KU Leuven, 3000 Leuven, Belgium
}

\section{A R T I C L E I N F O}

\section{Article history:}

Received 6 July 2012

Revised 12 October 2012

Accepted 2 November 2012

Available online 12 November 2012

\section{Keywords:}

ASD

BDNF

Behavior

CREB

Neurobeachin

Mouse

\begin{abstract}
A B S T R A C T
Neurobeachin (NBEA), a brain-enriched multidomain scaffolding protein involved in neurotransmitter release and synaptic functioning, has been identified as a candidate gene for autism spectrum disorder (ASD) in four unrelated patients haploinsufficient for NBEA. The aim of this study was to map the behavioral phenotype of $\mathrm{Nbea}^{+/-}$mice in order to understand its contribution to the pathogenesis of ASD. ASD-like behavioral variables of $\mathrm{Nbea}^{+/-}$mice were related to basal neuronal activity in different brain regions by in situ hybridizations and extracellular field recordings of synaptic plasticity in hippocampal cornu ammonis 1 (CA1) region. Levels of BDNF and phosphorylated cAMP response element-binding protein (CREB) were measured in an attempt to investigate putatively underlying changes in these neuromolecules. Nbea ${ }^{+/-}$mice exhibit several ASD-like features, including changes in self-grooming behavior, social behaviors, conditioned fear responses, and spatial learning and memory, which coincided with enhanced long-term potentiation (LTP) in their CA1 region. The observed alterations in learning and memory and hippocampal LTP are concomitant with decreased expression of the immediate early gene zif268 in dorsomedial striatum and hippocampal CA1 region, increased CREB phosphorylation, and increased hippocampal BDNF expression. These findings indicate that Nbea haploinsufficiency leads to various molecular and cellular changes that affect neuroplasticity and behavioral functions in mice, and could thus underlie the ASD symptomatology in NBEA deficient humans.
\end{abstract}

(C) 2012 Elsevier Inc. All rights reserved.

\section{Introduction}

With a prevalence of $0.6 \%$, autism spectrum disorders (ASDs) are among the most common neurodevelopmental disorders. ASD is characterized by various pathognomonic behavioral alterations including impaired social interaction and communication as well as frequent intellectual disability and anxiety (Fombonne, 2009). It is

\footnotetext{
* Corresponding author at: Laboratory of Biochemical Neuroendocrinology, Department of Human Genetics, KU Leuven, Gasthuisberg O\&N1, box 602, Herestraat 49, B-3000 Leuven, Belgium. Fax: + 3216346073 .

E-mail address: John.Creemers@med.kuleuven.be (J.W.M. Creemers).

Available online on ScienceDirect (www.sciencedirect.com).

1 Joint first author.

2 Joint last author.
}

generally accepted that there is a strong genetic basis for ASD that probably involves multiple genes (Abrahams and Geschwind, 2008). Recently, Neurobeachin (NBEA) has been suggested as an ASD candidate gene following the description of a patient with de novo balanced reciprocal translocation $t(5 ; 13)(\mathrm{q} 12.1 ; \mathrm{q} 13.2)$ with a breakpoint in intron 1 of NBEA (Volders et al., 2011). In addition, three patients with a monoallelic NBEA deletion were reported. Initially, a woman with autism was described with a deletion involving 13q13 and portions of q12 and q14. This deletion was not present in an autistic maternal cousin (Ritvo et al., 1988). In the second patient, a paternally derived deletion encompassed a $9 \mathrm{Mb}$ fragment in the 13q12-q13 region. The father was not further investigated for autistic features (Smith et al., 2002). A de novo deletion of 13q13.2-q14.1 was detected in a boy with autism in a screen for the incidence of cytogenetic abnormalities in a population of autism patients 
(Reddy, 2005). The NBEA gene is located in a $19 \mathrm{cM}$ region that was identified as a candidate region for ASD by linkage study and encompasses a low-frequency common fragile site (FRA13A) linked to ASD (Volders et al., 2011).

Little is known about the function of NBEA, but it has been shown to be a brain-enriched multidomain scaffolding protein located at tubulovesicular endomembranes near the trans-Golgi network (TGN) in neuronal soma and dendrites. A small fraction of NBEA is present at the postsynaptic membrane of a minority of synapses (Wang et al., 2000). The C-terminal part of NBEA contains a pleckstrin homology beige and Chediak-Higashi (BEACH) - WD40 domain module, generally implicated in vesicle trafficking (Gebauer et al., 2004; Jogl et al., 2002; Wang et al., 2000). The N-terminal region possesses a concanavalin A-like lectin domain flanked by Armadillo repeats suggested to play a role in intracellular trafficking (Breidenbach and Brunger, 2005; Burgess et al., 2009). Distal from these regions, an A kinase anchoring protein (AKAP) domain is present, recruiting cAMP-dependent protein kinase A (PKA) to endomembranes near the TGN by high affinity binding to its RII $\alpha$ subunit. NBEA has been shown to be involved in post-Golgi membrane trafficking and knockdown of Nbea in a neuroendocrine cell line led to enhanced secretion of dense-core secretory granules (DCSGs), the neuroendocrine counterpart of large dense-core vesicles (LDCVs) (Castermans et al., 2010). Furthermore, blood platelets of a patient haploinsufficient for NBEA showed abnormal morphology of dense granules (Castermans et al., 2010). Knockout Nbea in two independent mouse models resulted in perinatal lethality due to a complete block of evoked neuromuscular synaptic transmission (Medrihan et al., 2009; Su et al., 2004).

These findings suggest that Nbea haploinsufficiency may affect neural functioning in a subtle way, which could in fact explain the behavioral alterations observed in haploinsufficient patients. To test this hypothesis, we investigated whether Nbea haploinsufficiency in laboratory mice leads to changes in behavioral domains related to the diagnostic hallmarks of ASD.

We included tests of social behavior, since they relate to the core symptoms of ASD and only few ASD mouse models display such symptoms (Robertson and Feng, 2011), as well as tests for repetitive behavior, neuromotor performance, exploratory and general cage activity, sensitivity to auditory stimuli and sensorimotor gating, and learning and memory. Brain volume and regional neuronal activity were determined to examine whether Nbea haploinsufficiency affects gross brain morphology and function. As these analyses revealed changes in hippocampal activity, we assessed hippocampal (CA1) synaptic plasticity as well as some crucial plasticity-related molecular processes such as cAMP response element-binding protein (CREB) phosphorylation and levels of its target gene product brain-derived neurotrophic factor (BDNF), which might be at the basis of autistiform and neurocognitive defects.

\section{Materials and methods}

\section{Animals}

The GH240B transgenic line (Su et al., 2004) was backcrossed for at least 10 generations with C57BL/6JRj mice (Janvier). Female mice of 20 weeks of age (17 $\mathrm{Nbea}^{+/+}$and $18 \mathrm{Nbea}^{+/-}$mice) at the beginning of testing, were housed in groups in standard cages (wood-shaving bedding) under conventional laboratory conditions $\left(12 / 12 \mathrm{~h} \mathrm{light} / \mathrm{dark}\right.$ cycle, lights on at $\left.8 \mathrm{am} ; 22{ }^{\circ} \mathrm{C}\right)$ and were allowed food and water ad libitum. Group-housed females were used to avoid territorial aggression and fighting that frequently occurs in males. Behavioral experiments were conducted during the light phase of their activity cycle. All procedures were approved by the ethical research committee of KU Leuven. The grooming test was performed with a new group of 20 week old female mice ( $12 \mathrm{Nbea}^{+/+}$and 10 $\mathrm{Nbea}^{+/-}$mice). Body weight was monitored every 3 weeks. Body and brain weight were further examined in 12 week old female mice ( $n=10$ /group). Brains were isolated and immediately put on $-80{ }^{\circ} \mathrm{C}$ before weighing.

\section{Behavioral analysis}

Ambulatory cage activity, accelerating rotarod, PPI, open field test, elevated plus maze, social exploration test, SPSN test, contextual fear conditioning and MWM test were each conducted according to the protocol earlier described (Goddyn et al., 2006; Nadler et al., 2004; Naert et al., 2011; Paradee et al., 1999; Piccart et al., 2011; Van Dam et al., 2000). The behavioral grooming test was modified from the protocol described in Berridge (1989). A detailed description of all behavioral tests is available in the supplementary information (SI).

\section{In situ hybridizations for zif268}

As previously established, ISH for zif268 were performed on $25 \mu \mathrm{m}$ coronal cryostat sections ( $n=4 /$ genotype) (van Brussel et al., 2009). A detailed ISH protocol can be found in SI. Briefly, air-dried sections and a set of standard $\left[{ }^{14} \mathrm{C}\right]$ microscales (GE Healthcare, UK) were exposed to an autoradiographic film (Kodak, Belgium). Densitometric analysis of the digitized autoradiographic images was performed using the Image imaging system (National Institutes of Health, Bethesda, Maryland, USA). The optical density (OD) of each region of interest was expressed as mean gray value per pixel. OD measurements of prelimbic cortex (PL), anterior cingulate cortex (aCC), striatum, hippocampus and amygdala were determined in consecutive brain slices along the rostrocaudal axis, respectively from $+1.78 \mathrm{~mm}$ till $+1.54 \mathrm{~mm},+1.42 \mathrm{~mm}$ till $+1.10 \mathrm{~mm},+1.54 \mathrm{~mm}$ till $+1.10 \mathrm{~mm}$, $-1.22 \mathrm{~mm}$ to $-2.30 \mathrm{~mm}$ and $-1.46 \mathrm{~mm}$ to $-2.06 \mathrm{~mm}$ relative to bregma (Franklin, 2008). In the striatum, OD was determined for 3 subregions, dorsomedial (DMS), dorsolateral (DLS) and ventral striatum (VS), while in the hippocampus a detailed quantification of CA1 and CA3 was also performed.

\section{Electrophysiological recordings}

For electrophysiological experiments, 27- to 30-week-old female mice (same age as mice during MWM experiment) ( $n=6 /$ group) were killed by cervical dislocation. Details concerning the preparation of the transverse hippocampal slices and the recording conditions can be found in SI.

\section{RNA interference of Nbea in BTC3 cells}

The mouse pancreatic BTC3 cell line was grown in Dulbecco's modified Eagles medium supplemented with $10 \%$ fetal bovine serum at $37{ }^{\circ} \mathrm{C}, 5 \% \mathrm{CO}_{2}$. Lipofectamine RNAiMAX (Life Technologies, UK) was used to transiently transfect $\beta$ TC3 cells with either $40 \mathrm{nM}$ SiGENOME SMARTpool siRNA mouse Nbea or stealth RNAi siRNA negative control medium GC (Thermo Fisher Scientific, Illinois, USA). Cells were harvested after $72 \mathrm{~h}$ and divided for immunoblotting or determination of Nbea knockdown. The knockdown of Nbea was assessed by means of a quantitative PCR. RNA of transfected cells was extracted with Nucleospin RNA II (Macherey-Nagel, Germany) and cDNA synthesis was performed with iScript cDNA synthesis kit (Bio-Rad, California, USA). The mRNA expression of Nbea was calculated using the method of Livak with normalization to glyceraldehyde 3-phosphate dehydrogenase (GAPDH) as reference gene (Livak and Schmittgen, 2001). The following primers were used: Nbea_F 5' CTTTGTGCGGATCAACAGG 3'; Nbea_R 5' CATTGATGGAGTTGGCAAGA 3'; GAPDH_F 5' ATGGCCTTCCG TGTTCCT 3'; GAPDH_R 5' CAGGCGGCACGTCAGAT 3'. 


\section{Immunoblotting}

Standard Western blotting techniques were used to quantify CREB and PCREB relative to actin. For details concerning Western blot procedure see SI. Quantification was performed using Kodak Imager Software (Kodak, New York, USA) and the expression level in cells transfected with control siRNA was set at $100 \%$.

\section{Quantification of BDNF levels in hippocampus}

Hippocampi were dissected from 8 week old mice ( $n=7 /$ genotype) and immediately put on $-80{ }^{\circ} \mathrm{C}$. Tissue was homogenized in RIPA buffer $(25 \mathrm{mM}$ Tris- $\mathrm{HCl} \mathrm{pH} 7.6 ; 150 \mathrm{mM} \mathrm{NaCl} ; 1 \% \mathrm{NP}-40 ; 1 \%$ sodium deoxycholate; $0.1 \%$ SDS) containing a protease and phosphatase inhibitor cocktail (Roche, Belgium). BDNF levels were assessed by means of the Mouse BDNF ELISA kit (Abnova, Taiwan) according to manufacturer's instructions. Protein concentration was determined with BCA protein assay (Thermo Fisher Scientific, Illinois, USA) allowing normalization of BDNF content to total protein content.

\section{Statistical analysis}

Data are presented as mean with standard error of the mean (SEM). Significance of differences between mean values were analyzed using (where appropriate) two-tailed $t$ test, Mann-Whitney $U$ test (MWU), analysis of variance (ANOVA) or repeated measures (RM) ANOVA procedures with Tukey test post hoc comparison using Statistica version 9.0 (StatSoft Inc., Oklahoma, USA). All statistical tests were performed with 0.05 as the $\alpha$ level of significance $\left({ }^{*} P<0.05,{ }^{* *} P<0.01\right.$ and $\left.{ }^{* * *} P<0.001\right)$. The data from one excessively overweight $\mathrm{Nbea}^{+/+}$ mouse were excluded from the analyses.

\section{Results}

Increased relative brain volume in $\mathrm{Nbea}^{+1-}$ mice

The body weight of $\mathrm{Nbea}^{+/-}$mice was significantly lower than Nbea $^{+/+}$mice (RM-ANOVA: main effect of genotype: $F(1,32)=39.8$, $P<0.001)$ and this difference remained constant during behavioral testing (Fig. S1A). Further investigation in 12 week old female mice revealed reduced body and brain weight in $\mathrm{Nbea}^{+/-}$mice compared to Nbea $^{+/+}$mice $(t=9.80 ; P<0.001$ and $t=5.27 ; P<0.001$ respectively) resulting in an elevated brain to body weight ratio $(t=-8.60$; $P<0.001$ ) (Figs. S1B-D). Since $\mathrm{Nbea}^{+/-}$mice are small in stature, this was taken into account in all behavioral analyses.

Diminished ambulatory activity in $\mathrm{Nbea}^{+/-}$mice during night-day transition

Cage activity recording during a $23 \mathrm{~h}$ period showed an increase in activity for both groups shortly after introduction into the cage, but during the subsequent hours of testing, there was no significant difference between wildtype and heterozygous mice (RM-ANOVA: main effect of genotype: $F(1,32)=1.58, P=0.22$ ). However, RM-ANOVA showed a particular decrease in horizontal ambulatory cage activity in $\mathrm{Nbea}^{+/-}$mice compared to Nbea ${ }^{+/+}$mice during the sunrise period (RM-ANOVA: main effect of genotype: $F(1,32)=8.36, P=0.007$ ) (Fig. S2). The accelerating rotarod revealed no significant differences between $\mathrm{Nbea}^{+/-}$mice and $\mathrm{Nbea}^{+/+}$mice (RM-ANOVA: main effect of genotype: $F(1.32)=0.02, P=0.89)$. A learning curve was observed over the testing trials in both groups (RM-ANOVA: main effect of trial: $F(3,81)=9.44, P<0.001$ ) (Fig. S3).
$\mathrm{Nbea}^{+/+}$and $\mathrm{Nbea}^{+/-}$mice display similar exploratory activity

In the open field test, there was no significant difference between the total path length of $\mathrm{Nbea}^{+/-}$and $\mathrm{Nbea}^{+/+}$mice $(t=-1.72, P=0.096)$ (Fig. 1B). Both groups travelled more in the periphery compared to the center circle (main effect of zone: $F(1,64)=228.38 ; P<0.001$ ) and there were no significant differences between $\mathrm{Nbea}^{+/-}$and $\mathrm{Nbea}^{+/+}$ mice in this respect. Anxiety-related exploration in the elevated plus maze also failed to reveal differences between $\mathrm{Nbea}^{+/-}$and Nbea ${ }^{+/+}$ mice as both groups showed a preference for visiting the closed arms (main effect of arm: $F(1,32)=152.1, P<0.001$ ) and similar total beam crossings ( $t=1.09, P=0.29)$ (Fig. S4).

\section{Nbea $^{+/-}$mice show normal sensorimotor gating}

All mice exhibit normal auditory capacity except for one wildtype mouse that was excluded for further analysis. Habituation effects were observed across the three trial blocks in startle amplitude (RM-ANOVA: main effect of trial block: $F(2,62)=10.77, P<0.001$ ) but there was no effect of genotype $(F(1,31)=1.44, P=0.24)$ or trial block $\times$ genotype interaction $(F(2,62)=0.23, P=0.80)$ (Fig. S5A). Prepulse inhibition (PPI) revealed similar reactions of wildtype and heterozygous mice to all prepulses (RM-ANOVA: main effect of genotype: $F(1,31)=1.37$, $P=0.25)$ with a main effect of prepulse intensity $(F(4,124)=3.28$, $P=0.014$ ) (Fig. S5B).

\section{Increased self-grooming behavior of $\mathrm{Nbea}^{+/-}$mice}

As a measure for repetitive behavior, self-grooming was assessed after an aversive stimulus on the head of the test mouse. Heterozygous Nbea mice showed increased self-grooming behavior after their snout was sprayed with water mist compared to wildtype mice $(t=-3.49$; $P=0.002$ ) (Fig. 1A).

\section{Altered social behaviors in $\mathrm{Nbea}^{+/-}$mice}

In the social exploration test, the forced presence of two stranger mice in the center of the arena resulted in an enhanced overall ambulatory activity of $\mathrm{Nbea}^{+/-}$mice (main effect of genotype: $F(1,56)=16.1, P<0.001)$ leading to an increased path length in the center of the arena (post hoc comparison: $P=0.008$; Fig. 1C). Other approach measures were not different between the groups.

This indication of altered social behavior was further confirmed in the sociability and preference for social novelty (SPSN) test. In the first SPSN session, the social preference trial, the test mouse was given the choice to enter a side chamber containing an unfamiliar mouse (Stranger1) or a side chamber containing an empty cage. RM-ANOVA revealed a main effect of chamber $(F(1,32)=6.07, P=0.019)$ with wildtype mice exhibiting a preference for the chamber containing the unfamiliar mouse (Stranger1) over the empty cage (post hoc comparison: $P=0.014)$ whereas Nbea $^{+/-}$mice spent equal time in both chambers (Fig. 2A). Notably, during both sessions, the total distance travelled did not significantly differ between $\mathrm{Nbea}^{+/+}$and Nbea ${ }^{+/-}$ mice (session 1 : $U=124.5 ; P=0.51$ and session $2: U=126 ; P=0.55$ ). The actual social interaction of $\mathrm{Nbea}^{+/-}$mice with the stranger mouse, expressed as sniff time, only occurred during the first time period after exposure to the new chambers (post hoc comparison; $P<0.001$ ), whereas $\mathrm{Nbea}^{+/+}$mice interacted more with the stranger mouse during the first three time periods with significant post hoc comparison for time period $1(P=0.009)$ and time period $2(P=0.03)$. (Figs. 2B-C). During the second session of the test (the preference for social novelty trial), both $\mathrm{Nbea}^{+/-}$and $\mathrm{Nbea}^{+/+}$mice showed preference for the chamber containing the unfamiliar mouse (Stranger2) over the familiar mouse (Stranger1) (main effect of chamber: $F(1,32)=10.05$, $P=0.003$ with post hoc comparison for $\mathrm{Nbea}^{+/+}$mice: $P=0.048$ and Nbea $^{+/-}$mice: $P=0.021$ ) (Fig. 2D). Both wildtype and heterozygous 

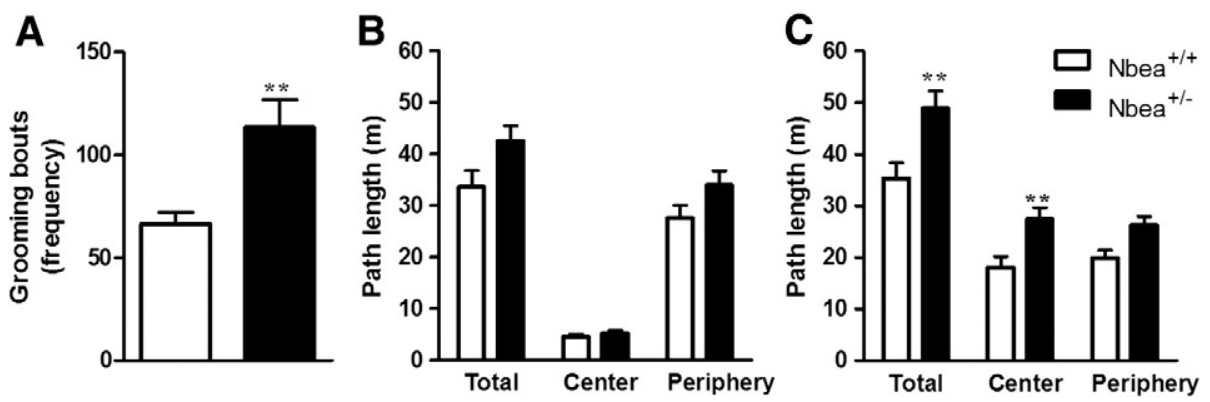

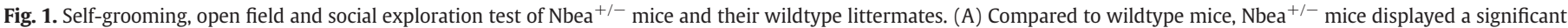

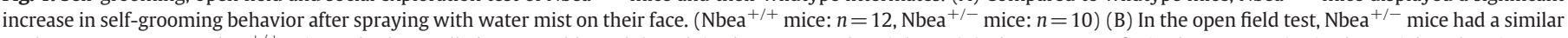

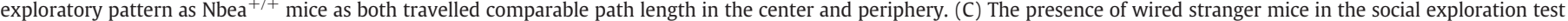
induced an increased overall exploration of the Nbea ${ }^{+/-}$mice leading to an increased exploration in the center and periphery. (Nbea ${ }^{+/+}$mice: $n=16$, Nbea ${ }^{+/-}$mice: $n=18$ ).

mice had increased sniff time with stranger 2 in the first 2 time periods with a significant post hoc comparison for $\mathrm{Nbea}^{+/+}$mice in the first time period $(P=0.022)$ (Figs. $2 \mathrm{E}-\mathrm{F})$.

Haploinsufficiency of Nbea enhances conditioned fear responses and impairs spatial learning and memory performance

In the contextual fear conditioning test, heterozygous mice showed overall increased percentage of freezing (RM-ANOVA: main effect of genotype: $F(1,32)=6276.7, P<0.001)$. When exposed to the same context after aversive footshock conditioning, Nbea ${ }^{+/-}$ mice displayed an increased percentage of freezing compared to Nbea $^{+/+}$mice (post hoc comparison: $P<0.001$ ) (Fig. 3). This difference could not be caused by increased baseline freezing behavior since there was no significant effect of genotype after initial exposure to the shock (post hoc comparison: $P=0.98$ ). Exposure to a new context (pre-CS) induced hardly any freezing, but presentation of the auditory stimulus resulted in a marked increase in percentage of freezing for both genotypes with no significant difference between wildtype and heterozygous mice (post hoc comparison: $P=0.12$ ) (Fig. 3).

During the acquisition trials of the Morris water maze (MWM) test, Nbea ${ }^{+/-}$mice travelled an overall increased distance which is represented as path length in Fig. 4A (RM-ANOVA: main effect of genotype: $F(1,32)=9.20 ; P=0.005)$. In the first probe trial, heterozygous mice failed to show a preference for the target quadrant compared to wildtype mice (interaction quadrant $\times$ genotype: $F(3,128)=2.78, P=0.044)$. This lack of spatial preference in the $\mathrm{Nbea}^{+/-}$group during the first probe trial was also illustrated by heat plots where the $\mathrm{Nbea}^{+/-}$mice demonstrates to search in 4 quadrants of the pool while $\mathrm{Nbea}^{+/+}$mice already showed preference for the platform quadrant (Fig. 4B). Notably, the reduced bodyweight of the Nbea ${ }^{+/-}$mice did not change their swimming velocity. In the second probe trial, performed after one more week of training, Nbea ${ }^{+/-}$mice did develop a preference for the target quadrant but less pronounced than $\mathrm{Nbea}^{+/+}$mice (interaction quadrant $\times$ genotype: $F(3,128)=4.74 ; P=0.0036$ with post hoc comparison: $P=0.020$ ) (Fig. $4 \mathrm{~B}$ ).
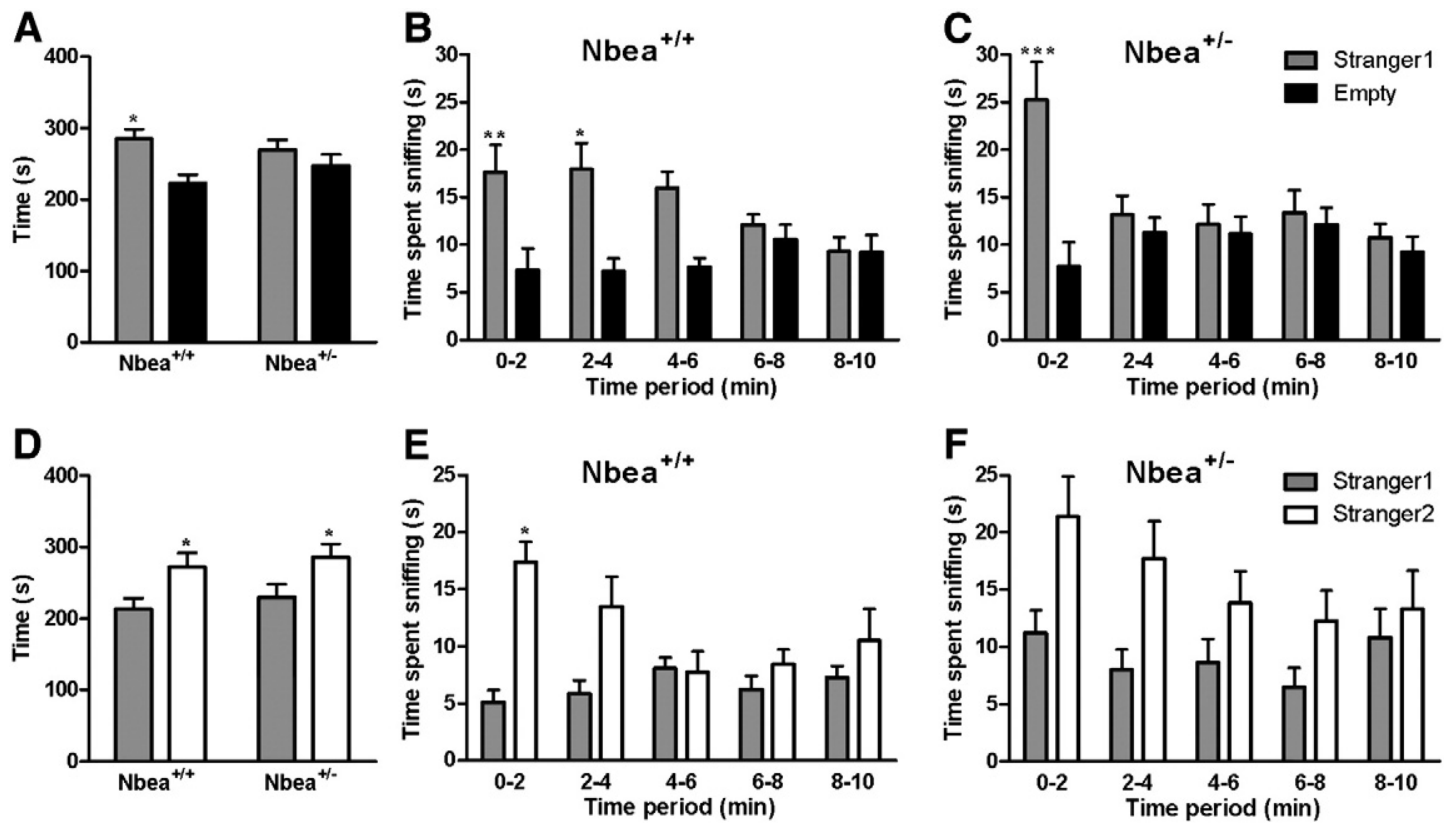

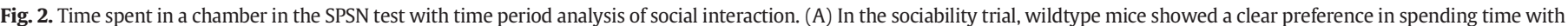

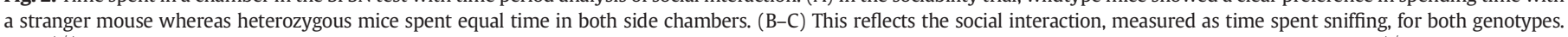

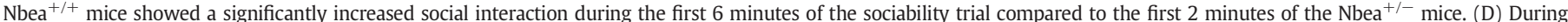

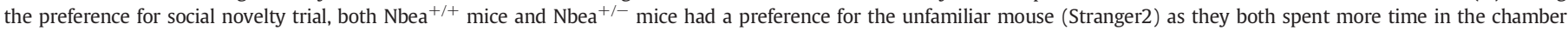

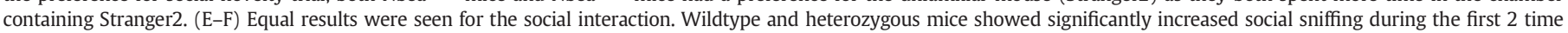
periods. (Nbea ${ }^{+/+}$mice: $n=16$, Nbea $^{+/-}$mice: $n=18$ ). 


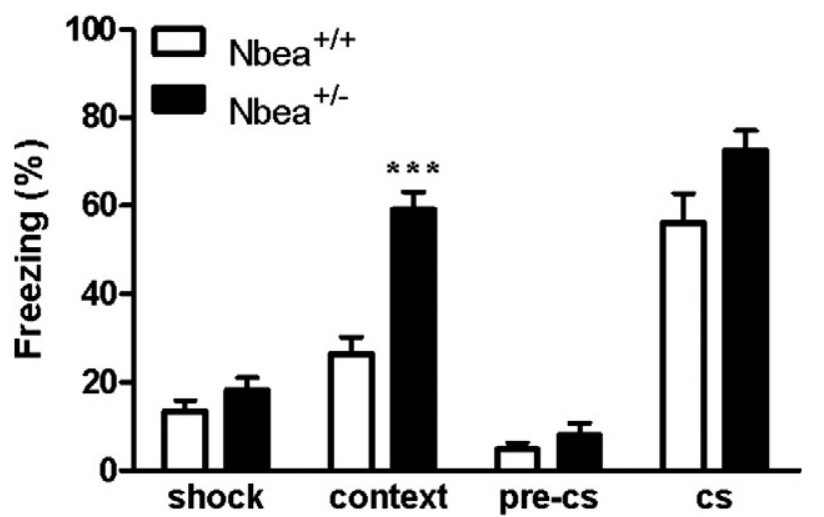

Fig. 3. Contextual fear conditioning test of wildtype and heterozygous mice. Nbea ${ }^{+/-}$ mice showed significant increased percentage of freezing after exposure to the same context. This cannot be explained by an increased baseline freezing behavior since no significant difference of freezing in the shock condition was present. In the conditioning phase, Nbea ${ }^{+/-}$mice displayed a tendency for higher freezing behavior after hearing the conditioned stimulus however the post hoc comparison was not significant. This tendency was not due to a shift in baseline freezing because no significant difference in freezing behavior was observed in the pre-conditioned stimulus phase. (Nbea $^{+/+}$mice: $n=16$, Nbea $^{+/-}$mice: $n=18$ ).

Decreased expression of zif268 mRNA in the dorsomedial striatum and hippocampal CA1 of $\mathrm{Nbea}^{+/-}$mice

The expression level of zif268 mRNA was decreased in DMS of Nbea $^{+/-}$mice $(t=2.35, P=0.020)$ (Figs. $\left.5 \mathrm{~A}, \mathrm{C}\right)$, but no significant difference was detected for DLS and VS (Fig. S6A). In addition, reduced expression levels of zif268 mRNA in the total hippocampal formation $(t=2.91, P=0.005)$ as well as a marked decrease in expression in the CA1 region $(t=2.17, P=0.033)$ but not in CA3 region was found in Nbea $^{+/-}$mice (Figs. 5B-C and S6B). Expression of zif268 in medial prefrontal cortex (PL and $\mathrm{aCC}$ ) and amygdala did not differ between $\mathrm{Nbea}^{+/-}$and Nbea ${ }^{+/+}$mice (Fig. S6C-D).

Enhanced long-term potentiation in CA1 area of the hippocampus in Nbea $^{+1-}$ mice

Extracellular recordings in the CA1 area of hippocampus indicated normal basic synaptic excitability in $\mathrm{Nbea}^{+/-}$mice since the I/O curves did not differ between $\mathrm{Nbea}^{+/+}$mice and Nbea ${ }^{+/-}$mice (Fig. 6A). Likewise, $\mathrm{Nbea}^{+/-}$mice did not show alterations in presynaptically mediated short-term plasticity as proven by similar ratios of PPF as compared to their wildtype littermates (Fig. 6B). LTP, however, was evidently improved. Slices of $\mathrm{Nbea}^{+/-}$mice attained a significantly higher initial magnitude of potentiation as compared to $\mathrm{Nbea}^{+/+}$mice ( 1 min: Nbea $^{+/-}$mice: $264.0 \pm 27.8 \%$; Nbea $^{+/+}$mice: $185.0 \pm 25.2 \%$, MWU: $P=0.034)$. Since the decay of potentiation was similar in both genotypes this resulted in a main effect of genotype (RM-ANOVA: $F(1,14)=4.62 ; P=0.04$ ) (Fig. $6 C$ ).

Nbea deficiency leads to increased phosphorylation of CREB in vitro and higher BDNF levels in vivo

Another mouse model that displayed exorbitant LTP combined with a suboptimal performance in the MWM task is the transgenic VP16-CREB mouse, a model for constitutively active CREB (Viosca et al., 2009). CREB is activated upon phosphorylation of serine 133 , a demonstrated target-site of PKA (Gonzalez and Montminy, 1989). Since Nbea is an AKAP protein and defective sequestering of inactive PKA has been shown to result in increased PKA activity (Corstens et al., 2006) this might lead to changes in the pCREB/CREB ratio. Therefore, the phosphorylation status of CREB was examined in the neuroendocrine cell line $\beta$ TC3, after knockdown of Nbea. The expression of Nbea was reduced with $42 \% \pm 5.8 \%$ which resembles the
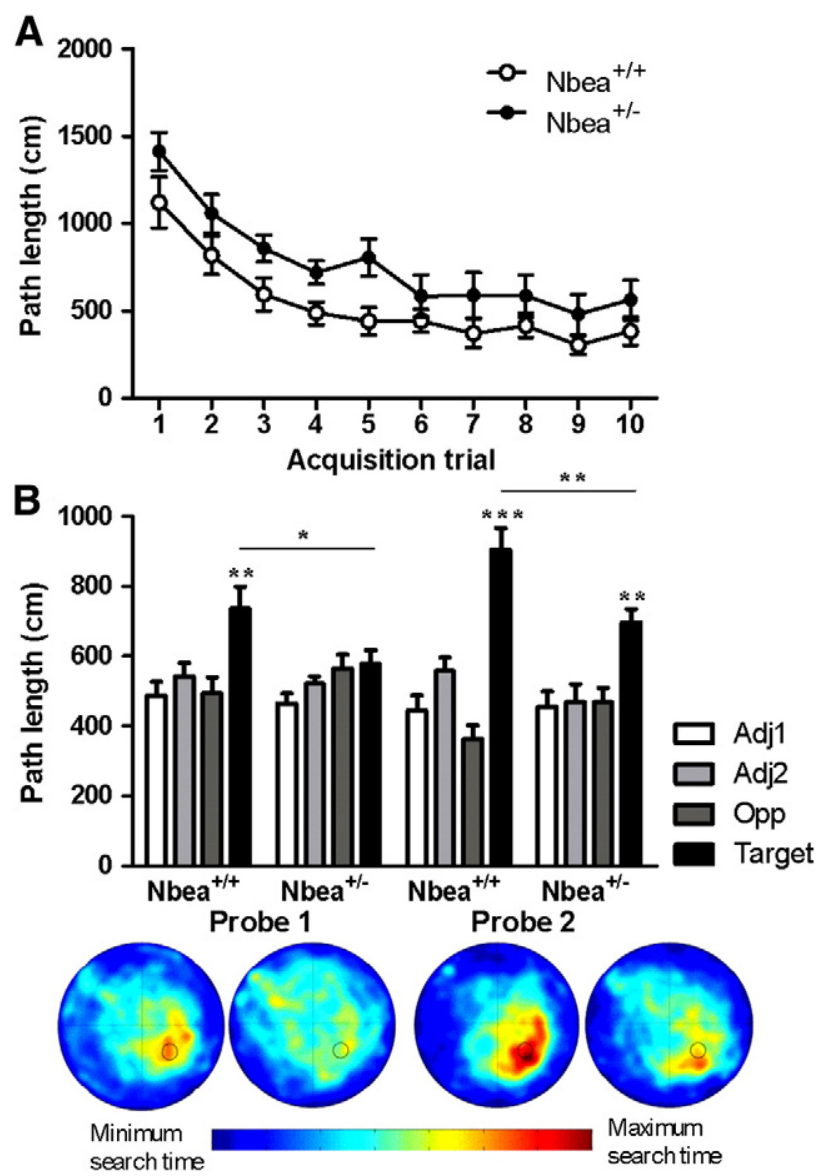

Fig. 4. Learning and memory assessment in the Morris water maze test. (A) During the acquisition trials, Nbea ${ }^{+/-}$mice travelled increased path length in order to find the hidden platform as detected by RM-ANOVA but post hoc comparison revealed no significant difference. (B) In the first probe trial, wildtype mice were able to successfully find the location of the platform, measured as distance covered in the target quadrant, whereas $\mathrm{Nbea}^{+/-}$mice did not show a preference for the target quadrant. In the second probe trial both $\mathrm{Nbea}^{+/+}$ mice and $\mathrm{Nbea}^{+/-}$mice swam more in the target quadrant but wildtype mice were more efficient in locating the hidden platform. Heat plots per genotype per probe trial indicate the time each group spent on a specific position with a warmer color for longer time periods. These heat plots confirmed that $\mathrm{Nbea}^{+/-}$mice were not able to locate the platform in the first probe trial. In probe trial $2, \mathrm{Nbea}^{+/-}$mice had developed a preference in spending time in the target quadrant but to lesser extent than $\mathrm{Nbea}^{+/+}$mice. The heat plots illustrated that the performance of $\mathrm{Nbea}^{+/-}$mice in the second probe trial was of the same level as $\mathrm{Nbea}^{+/+}$mice in the first probe trial. (Nbea ${ }^{+/+}$mice: $n=16$, Nbea $^{+/-}$mice: $n=18$ ).

haploinsufficient status of Nbea ${ }^{+/-}$mice. Quantitative analysis of 3 independent experiments indicated that Nbea haploinsufficiency did not alter the total amount of CREB $(t=0.87 ; P=0.43)$ but significantly increased the phosphorylation of CREB (factor 2.35) $(t=-4.02 ; P=0.016)$ (Fig. 7A). Several attempts to extrapolate these data to the hippocampus failed because pCREB was too low for direct detection and quantification. To overcome this issue, the levels of BDNF were determined in 8 week old hippocampi as BDNF is a target of pCREB (Barco et al., 2005) and reaches the highest expression level at the age of 8 weeks. The hippocampus of $\mathrm{Nbea}^{+/-}$mice contained $32 \%$ more BDNF compared to Nbea ${ }^{+/+}$mice $(t=-2.28 ; P=0.041)$ which is indicative for a higher $\mathrm{pCREB} / \mathrm{CREB}$ ratio in the hippocampus (Fig. 7B).

\section{Discussion}

$\mathrm{Nbea}^{+/-}$mice displayed several phenotypic changes directly or indirectly related to ASD symptoms. Haploinsufficiency of Nbea increased self-grooming behavior, decreased sociability read-outs, enhanced conditioned fear, and impaired spatial learning and memory. These findings coincided with increased relative brain volume and 


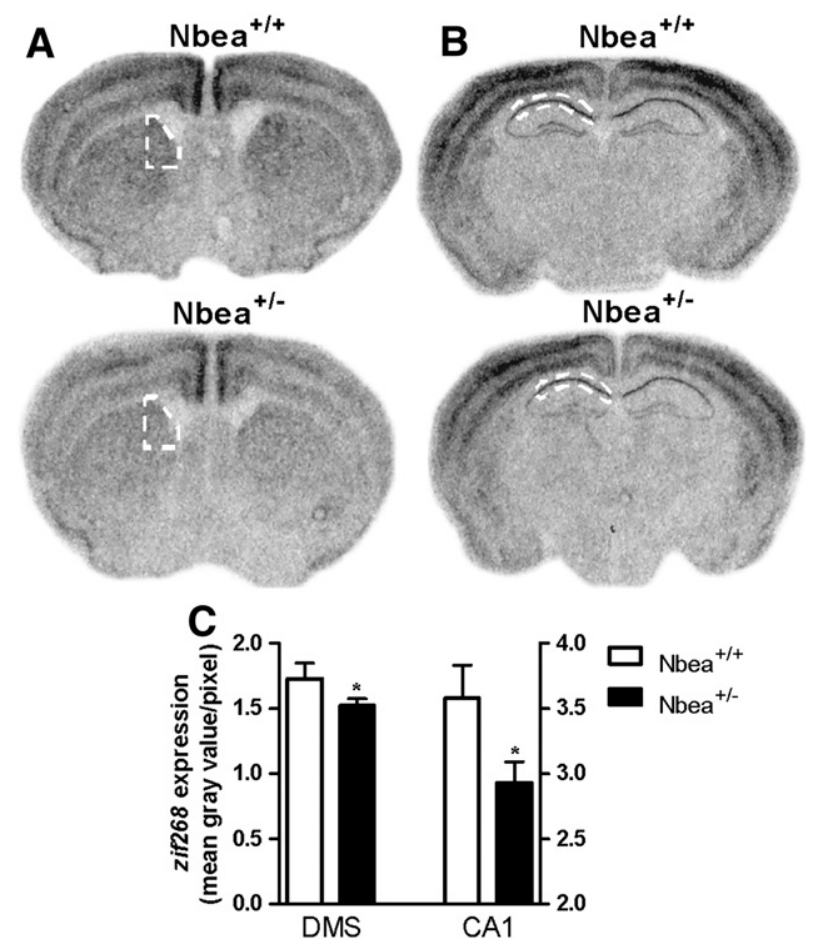

Fig. 5. In situ hybridizations for zif268 in striatum and hippocampus. (A) Brain sections of $\mathrm{Nbea}^{+/+}$mice and $\mathrm{Nbea}^{+/-}$mice with the DMS indicated with a dotted line. (B) Brain sections of $\mathrm{Nbea}^{+/+}$mice and $\mathrm{Nbea}^{+/-}$mice with the CA1 region of the hippocampus indicated with a dotted line. (C) Quantification of zif268 expression by Image imaging system. Both DMS and CA1 region of $\mathrm{Nbea}^{+/-}$mice had a decreased expression of zif268 indicating a decreased neuronal activity in those regions. (Nbea $^{+/+}$mice: $n=4$, Nbea $^{+/-}$mice: $n=4$ ).

alterations in DMS and hippocampus activation (based on zif268 ISH) and enhanced hippocampal LTP. At the molecular level, Nbea knockdown in BTC3 cells resulted in increased phosphorylation of CREB, and higher levels of its target gene product BDNF in the hippocampus of $\mathrm{Nbea}^{+/-}$mice.

Prepulse inhibition is commonly used to test sensorimotor gating, which is often impaired in patients with ASD or schizophrenia (Perry et al., 2007). We failed to observe any defect but C57BL/6J mice have one of the lowest PPI levels, which complicates the detection of impairments (Crawley et al., 1997).

Several other behavioral tests showed changes in $\mathrm{Nbea}^{+/-}$mice that might relate to characteristic behavioral symptoms of ASD, especially those of patients with NBEA deficiency (Castermans et al., 2003). $\mathrm{Nbea}^{+/-}$mice showed an increase in self-grooming behavior after their snout was sprayed with water mist. Tests of repetitive behavior are often executed in the context of obsessive-compulsive disorder or ASD (Wang et al., 2011; Welch et al., 2007). Increased self-grooming behavior of $\mathrm{Nbea}^{+/-}$mice might be indicative for impairments in repetitive behavior (Silverman et al., 2010). Moreover, Nbea ${ }^{+/-}$mice displayed changes during social exploration and the SPSN test. Sociability read-outs of the SPSN task might be the most relevant autism-like symptoms in mice (Yang et al., 2011). Therefore, diminished sociability in Nbea $^{+/-}$mice may relate to social approach deficits of autistic patients (Silverman et al., 2010). Reduced sociability could not be attributed to changed exploration, neuromotor performance, or anxiety or innate fear, since $\mathrm{Nbea}^{+/-}$mice performed normally in open field, rotarod and elevated plus maze tests (Moy et al., 2009). Although Nbea $^{+/-}$mice show defects in social approach and social exploration, their preference for social novelty trial was normal. Thus, $\mathrm{Nbea}^{+/-}$mice are able to distinguish a novel mouse from a previously encountered mouse, which is reminiscent of behavioral changes in other ASD mouse models.
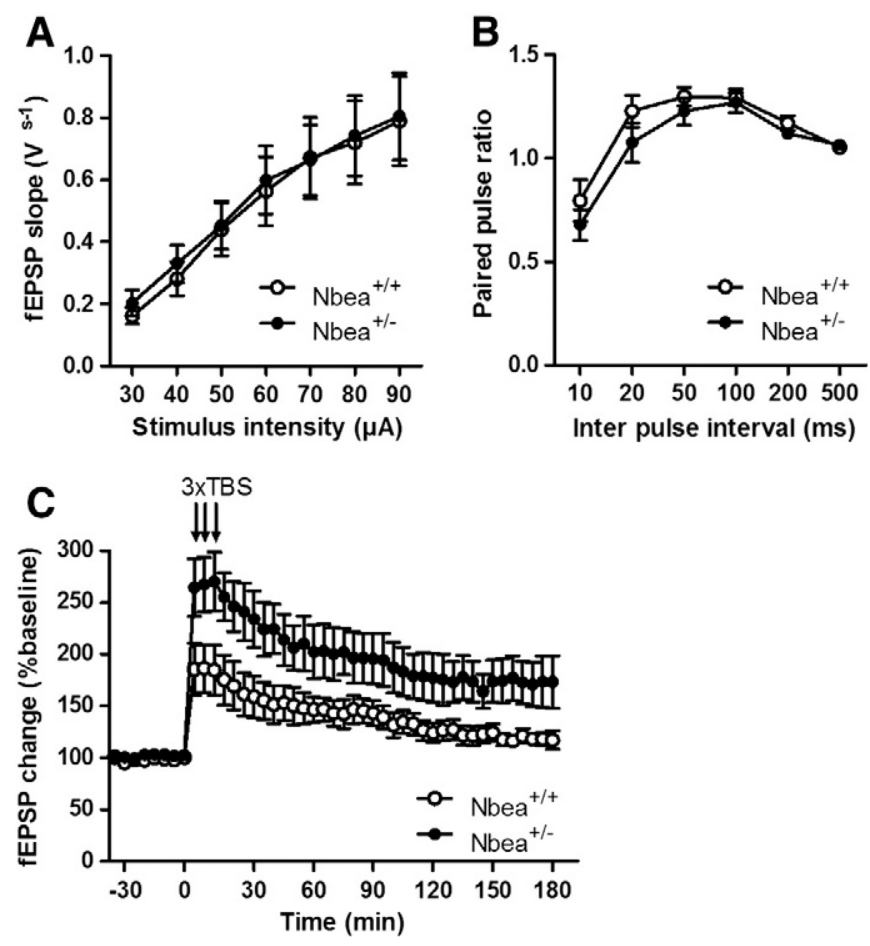

Fig. 6. Electrophysiological recordings in CA1 of the hippocampus. (A) Based on $I / O$ curves, basic synaptic synaptic excitability was normal in $\mathrm{Nbea}^{+/-}$mice at all stimulus intensities measured (Nbea ${ }^{+/-}$mice: $n=7$; $\mathrm{Nbea}^{+/+}$mice $n=9$ ) (B) PPF did not differ between $\mathrm{Nbea}^{+/+}$and $\mathrm{Nbea}^{+/-}$mice, regardless of the inter pulse interval, indicating normal short-term presynaptic plasticity ( $\mathrm{Nbea}^{+/-}$mice: $n=8$; $\mathrm{Nbea}^{+/+}$mice $n=9$ ). (C) LTP was significantly increased in $\mathrm{Nbea}^{+/-}$mice after $3 \times$ TBS detected by RM-ANOVA. (Nbea ${ }^{+/+}$mice $n=9$, bea $^{+/-}$mice: $n=7$ ).

Increased brain/body weight ratio is a common feature of ASD present in approximately 20\% of ASD individuals (McCaffery and Deutsch, 2005; Stigler et al., 2011). Although $\mathrm{Nbea}^{+/-}$mice are smaller in stature due to selective inhibition of postnatal pituitary development (Su et al., 2004), this cannot account for the increased relative brain volume as growth hormone deficiency generally leads to a decreased brain volume (Segal et al., 2004). Of notice, Olszewski et al. (2012) observed no dwarfism in Nbea ${ }^{+/-}$mice generated by means of the gene-trap vector system (Olszewski et al., 2012). This discrepancy might be due to the different methods used to generate the mouse models. A possible hypothesis of the basis of the detected brain overgrowth is the elevated BDNF level in the hippocampus as mature BDNF regulates neuronal structure whereas the precursor proBDNF mediates apoptosis (Lee et al., 2001). The elevated level of BDNF in the hippocampus might be indicative for an overall increased level of BDNF. In vitro, Nbea has been identified as a negative regulator of the regulated secretion (Castermans et al., 2010). Defects in fine-tuning of vesicular secretion could lead to increased blood levels of BDNF, which has been reported in several patients and mouse models for ASD (Nickl-Jockschat and Michel, 2011). Moreover, NBEA has recently been identified in a screen for proteins important for neuronal differentiation (Iwaniuk et al., 2010).

No differences in zif268 expression were detected in aCC and amygdala, two regions known to be important for social processing, but we did identify reduced expression in hippocampus and DMS. Defects in zif268-controlled signaling in hippocampus and striatum are likely to affect hippocampus-prefrontal (the extensive connections between the ventral part of aCC and hippocampus) and corticostriatal pathways that are crucial for social behavior as well as various neurocognitive functions. Recently, it has been shown that Nbea is required for dendritic spine formation, likely by regulating the assembly of F-actin (Niesmann et al., 2011). These findings were established in 
A
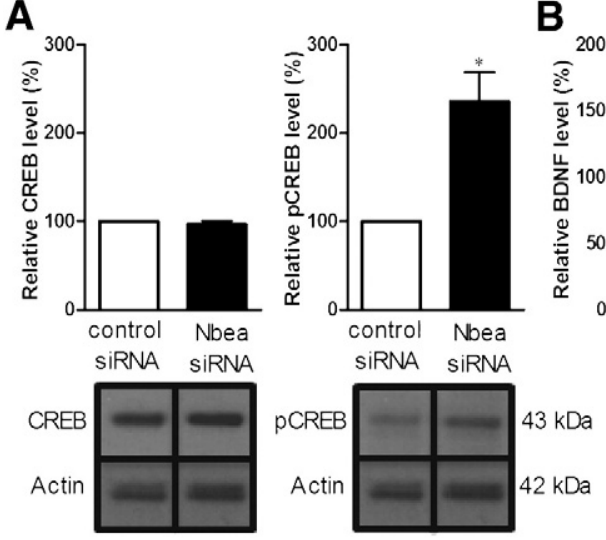

Fig. 7. Measurement of phosphorylation of CREB in vitro and BDNF levels in vivo. (A) Total amount of CREB in $\beta T C 3$ cells was unaltered after knockdown of Nbea whereas the proportion of pCREB increased with factor 2.35. Levels of CREB and PCREB were normalized first against actin and the expression level in cells transfected with control siRNA was set at $100 \%(n=3)$. (B) The BDNF level was first normalized to the total protein content of the sample and BDNF level of wildtype hippocampi was set at $100 \%$. The BDNF levels in heterozygous hippocampi were $32 \%$ higher. ( $\mathrm{Nbea}^{+/+}$mice $n=7$, $\mathrm{Nbea}^{+/-}$mice: $n=7$ ).

cultures of cortical neurons from $\mathrm{Nbea}^{+/-}$mice, providing further evidence for defective corticostriatal signaling. In addition, Nbea has been suggested to be involved in plasticity of the visual cortex (Rietman et al., 2012).

Nbea $^{+/-}$mice clearly showed impaired learning and memory in MWM test. They displayed delayed spatial learning, as they were unable to find the hidden platform during the first probe trial, but succeeded in locating it during the second probe trial. Apparently, $\mathrm{Nbea}^{+/-}$mice were able to overcome these deficits in spatial learning ability at least partly with prolonged training. Impaired but partially preserved learning and memory abilities were also found in other ASD models (Robertson and Feng, 2011), and spatial working memory is impaired in adolescents and adults with ASD (Williams et al., 2005).

Changes in hippocampal LTP could explain the poor performance of $\mathrm{Nbea}^{+/-}$mice in the MWM test. Accumulating evidence suggests that enhanced LTP can have detrimental consequences on learning and memory performance (D'Hooge et al., 2005; Jolas et al., 2002; Nguyen et al., 2000; Parent et al., 1999) and hippocampal place-cell functions (Taverna et al., 2005). VP16-CREB mice with constitutively active CREB, also displayed normal I/O curves and PPF with enhanced LTP and suboptimal learning in MWM task (Barco et al., 2002; Viosca et al., 2009). Faciliation of LTP might result in an unfavorable shift of synaptic weights in neuronal networks during learning, saturating these networks and causing reduced learning capacity (Lopez de Armentia et al., 2007; Viosca et al., 2009). We found that Nbea ${ }^{+/-}$mice also showed enhanced context-dependent fear responses. The change in learned fear could again be part of the ASD-like phenotype of $\mathrm{Nbea}^{+/-}$mice. Two mouse lines with moderate levels of constitutively active CREB showed enhanced context freezing in a comparable protocol for aversive footshock conditioning (Suzuki et al., 2011). This suggests that increased pCREB levels might be at the basis of the learning and memory defects in $\mathrm{Nbea}^{+/-}$mice.

$\mathrm{Nbea}^{+/-}$mice displayed reduced levels of zif268 in hippocampal CA1 and DMS, which are two brain regions crucial for spatial learning and memory (D'Hooge and De Deyn, 2001; Dillon et al., 2008). This change in levels of zif268, an immediate early gene in spatial memory processes (Guzowski et al., 2001), could contribute to the learning and memory defects observed here. While pCREB plays an important role in induction and maintenance of LTP, zif268 plays a critical role in the stabilization of late LTP (Bozon et al., 2003; Jones et al., 2001), and zif268 knockout mice were unable to maintain stable place fields in CA1 region (Renaudineau et al., 2009). Both pCREB and the downstream expression of zif268 are required in hippocampus and striatum for spatial memory formation (Porte et al., 2008). Furthermore, disturbed hippocampal or striatal signaling can be consistent with changes in learned fear since enhanced or exaggerated fear responses have been observed in juvenile mice, and attributed to immaturity of hippocampus-prefrontal pathways (Ito et al., 2009). CREB activation probably has a key role in delineating these learning-dependent circuits, and the decreased zif268 levels in DMS and hippocampus of $\mathrm{Nbea}^{+/-}$mice may have resulted from increased CREB phosphorylation.

\section{Acknowledgments}

We thank Sandra Meulemans and Leen Van Aerschot the technical assistance. This work was supported by the "agentschap voor innovatie door wetenschap en technologie Vlaanderen (IWT-Vlaanderen)", "steunfonds Marguerite-Marie Delacroix", KU Leuven [IDO/08/ 013, GOA/12/008], Methusalem grant of the Flemish Government and MEMOSAD [F2-2007-200611] of the European Union. The funding sources had no involvement in the study design; the collection, analysis and interpretation of data; in writing the report and the decision to submit the article for publication.

\section{Appendix A. Supplementary data}

Supplementary data to this article can be found online at http:// dx.doi.org/10.1016/j.nbd.2012.11.004.

\section{References}

Abrahams, B., Geschwind, D., 2008. Advances in autism genetics: on the threshold of a new neurobiology. Nat. Rev. Genet. 9, 341-355.

Barco, A., et al., 2002. Expression of constitutively active CREB protein facilitates the late phase of long-term potentiation by enhancing synaptic capture. Cell 108 , 689-703.

Barco, A., et al., 2005. Gene expression profiling of facilitated L-LTP in VP16-CREB mice reveals that BDNF is critical for the maintenance of LTP and its synaptic capture. Neuron 48, 123-137.

Berridge, K.C., 1989. Progressive degradation of serial grooming chains by descending decerebration. Behav. Brain Res. 33, 241-253.

Bozon, B., et al., 2003. MAPK, CREB and zif268 are all required for the consolidation of recognition memory. Philos. Trans. R. Soc. Lond. B Biol. Sci. 358, 805-814.

Breidenbach, M., Brunger, A., 2005. New insights into clostridial neurotoxin-SNARE interactions. Trends Mol. Med. 11, 377-381.

Burgess, A., et al., 2009. A concanavalin A-like lectin domain in the CHS1/LYST protein, shared by members of the BEACH family. Bioinformatics 25, 1219-1222.

Castermans, D., et al., 2003. The neurobeachin gene is disrupted by a translocation in a patient with idiopathic autism. J. Med. Genet. 40, 352-356.

Castermans, D., et al., 2010. SCAMP5, NBEA and AMISYN: three candidate genes for autism involved in secretion of large dense-core vesicles. Hum. Mol. Genet. 19, 1368-1378.

Corstens, G.J., et al., 2006. The effects of disruption of A kinase anchoring proteinprotein kinase A association on protein kinase A signalling in neuroendocrine melanotroph cells of Xenopus laevis. J. Neuroendocrinol. 18, 477-483.

Crawley, J.N., et al., 1997. Behavioral phenotypes of inbred mouse strains: implications and recommendations for molecular studies. Psychopharmacology (Berl) 132 $107-124$.

D'Hooge, R., De Deyn, P.P., 2001. Applications of the Morris water maze in the study of learning and memory. Brain Res. Brain Res. Rev. 36, 60-90.

D'Hooge, R., et al., 2005. Neurocognitive and psychotiform behavioral alterations and enhanced hippocampal long-term potentiation in transgenic mice displaying neuropathological features of human alpha-mannosidosis. J. Neurosci. 25, 6539-6549.

Dillon, G.M., et al., 2008. Excitotoxic lesions restricted to the dorsal CA1 field of the hippocampus impair spatial memory and extinction learning in C57BL/6 mice. Neurobiol. Learn. Mem. 90, 426-433.

Fombonne, E., 2009. Epidemiology of pervasive developmental disorders. Pediatr. Res. 65, 591-598.

Franklin, K.B., Paxinos, G., 2008. The Mouse Brain in Stereotaxic Coordinates. Academic Press, San Diego.

Gebauer, D., et al., 2004. Crystal structure of the PH-BEACH domains of human LRBA/BGL Biochemistry 43, 14873-14880.

Goddyn, H., et al., 2006. Differences in behavioural test battery performance between mice with hippocampal and cerebellar lesions. Behav. Brain Res. 173, 138-147.

Gonzalez, G.A., Montminy, M.R., 1989. Cyclic AMP stimulates somatostatin gene transcription by phosphorylation of CREB at serine 133. Cell 59, 675-680

Guzowski, J.F., et al., 2001. Experience-dependent gene expression in the rat hippocampus after spatial learning: a comparison of the immediate-early genes Arc, c-fos, and zif268. J. Neurosci. 21, 5089-5098. 
Ito, W., et al., 2009. Enhanced generalization of auditory conditioned fear in juvenile mice. Learn. Mem. 16, 187-192.

Iwaniuk, K.M., et al., 2010. Network-like impact of MicroRNAs on neuronal lineage differentiation of unrestricted somatic stem cells from human cord blood (USSC). Stem Cells Dev.

Jogl, G., et al., 2002. Crystal structure of the BEACH domain reveals an unusual fold and extensive association with a novel PH domain. EMBO J. 21, 4785-4795.

Jolas, T., et al., 2002. Long-term potentiation is increased in the CA1 area of the hippocampus of APP(swe/ind) CRND8 mice. Neurobiol. Dis. 11, 394-409.

Jones, M.W., et al., 2001. A requirement for the immediate early gene Zif268 in the expression of late LTP and long-term memories. Nat. Neurosci. 4, 289-296.

Lee, R., et al., 2001. Regulation of cell survival by secreted proneurotrophins. Science 294, 1945-1948.

Livak, K.J., Schmittgen, T.D., 2001. Analysis of relative gene expression data using real-time quantitative PCR and the 2(-Delta Delta C(T)) Method. Methods 25, 402-408.

Lopez de Armentia, M., et al., 2007. cAMP response element-binding protein-mediated gene expression increases the intrinsic excitability of CA1 pyramidal neurons. J. Neurosci. 27, 13909-13918.

McCaffery, P., Deutsch, C.K., 2005. Macrocephaly and the control of brain growth in autistic disorders. Prog. Neurobiol. 77, 38-56.

Medrihan, L., et al., 2009. Neurobeachin, a protein implicated in membrane protein traffic and autism, is required for the formation and functioning of central synapses. J. Physiol.

Moy, S.S., et al., 2009. Social approach in genetically engineered mouse lines relevant to autism. Genes Brain Behav. 8, 129-142.

Nadler, J.J., et al., 2004. Automated apparatus for quantitation of social approach behaviors in mice. Genes Brain Behav. 3, 303-314.

Naert, A., et al., 2011. Nocturnal hyperactivity, increased social novelty preference and delayed extinction of fear responses in post-weaning socially isolated mice. Brain Res. Bull. 85, 354-362.

Nguyen, P.V., et al., 2000. Strain-dependent differences in LTP and hippocampusdependent memory in inbred mice. Learn. Mem. 7, 170-179.

Nickl-Jockschat, T., Michel, T.M., 2011. The role of neurotrophic factors in autism. Mol. Psychiatry 16, 478-490.

Niesmann, K., et al., 2011. Dendritic spine formation and synaptic function require neurobeachin. Nat. Commun. 2, 557.

Olszewski, P.K., et al., 2012. Neurobeachin, a regulator of synaptic protein targeting, is associated with body fat mass and feeding behavior in mice and body-mass index in humans. PLoS Genet. 8, e1002568.

Paradee, W., et al., 1999. Fragile X mouse: strain effects of knockout phenotype and evidence suggesting deficient amygdala function. Neuroscience 94, 185-192.

Parent, A., et al., 1999. Synaptic transmission and hippocampal long-term potentiation in transgenic mice expressing FAD-linked presenilin 1. Neurobiol. Dis. 6, 56-62.

Perry, W., et al., 2007. Sensorimotor gating deficits in adults with autism. Biol. Psychiatry 61, 482-486.

Piccart, E., et al., 2011. Impaired appetitively as well as aversively motivated behaviors and learning in PDE10A-deficient mice suggest a role for striatal signaling in evaluative salience attribution. Neurobiol. Learn. Mem. 95, 260-269.
Porte, Y., et al., 2008. Spatial memory in the Morris water maze and activation of cyclic AMP response element-binding (CREB) protein within the mouse hippocampus. Learn. Mem. 15, 885-894.

Reddy, K., 2005. Cytogenetic abnormalities and fragile-X syndrome in autism spectrum disorder. BMC Med. Genet. 6, 3

Renaudineau, S., et al., 2009. Impaired long-term stability of CA1 place cell representation in mice lacking the transcription factor zif268/egr1. Proc. Natl. Acad. Sci. U. S. A. 106, 11771-11775.

Rietman, M.L., et al., 2012. Candidate genes in ocular dominance plasticity. Front. Neurosci. $6,11$.

Ritvo, E., et al., 1988. Association of autism, retinoblastoma, and reduced esterase D activity. Arch. Gen. Psychiatry 45, 600.

Robertson, H.R., Feng, G., 2011. Annual Research Review: transgenic mouse models of childhood-onset psychiatric disorders. J. Child Psychol. Psychiatry 52, 442-475.

Segal, D.G., et al., 2004. Craniofacial and acral growth responses in growth hormonedeficient children treated with growth hormone. J. Pediatr. 144, 437-443.

Silverman, J.L., et al., 2010. Behavioural phenotyping assays for mouse models of autism. Nat. Rev. Neurosci. 11, 490-502.

Smith, M., et al., 2002. Molecular genetic delineation of a deletion of chromosome $13 \mathrm{q} 12 \rightarrow \mathrm{q} 13$ in a patient with autism and auditory processing deficits. Cytogenet. Genome Res. 98, 233-239.

Stigler, K.A., et al., 2011. Structural and functional magnetic resonance imaging of autism spectrum disorders. Brain Res. 1380, 146-161.

$\mathrm{Su}, \mathrm{Y}$., et al., 2004. Neurobeachin is essential for neuromuscular synaptic transmission. J. Neurosci. 24, 3627-3636.

Suzuki, A., et al., 2011. Upregulation of CREB-mediated transcription enhances both short- and long-term memory. J. Neurosci. 31, 8786-8802.

Taverna, F.A., et al., 2005. Defective place cell activity in nociceptin receptor knockout mice with elevated NMDA receptor-dependent long-term potentiation. J. Physiol. 565, 579-591.

van Brussel, L., et al., 2009. Identification and localization of functional subdivisions in the visual cortex of the adult mouse. J. Comp. Neurol. 514, 107-116.

Van Dam, D., et al., 2000. Spatial learning, contextual fear conditioning and conditioned emotional response in Fmr1 knockout mice. Behav. Brain Res. 117, 127-136.

Viosca, J., et al., 2009. Chronic enhancement of CREB activity in the hippocampus interferes with the retrieval of spatial information. Learn. Mem. 16, 198-209.

Volders, K., et al., 2011. The autism candidate gene Neurobeachin encodes a scaffolding protein implicated in membrane trafficking and signaling. Curr. Mol. Med. 11, 204-217.

Wang, X, et al., 2000. Neurobeachin: A protein kinase A-anchoring, beige/Chediakhigashi protein homolog implicated in neuronal membrane traffic. J. Neurosci. $20,8551-8565$

Wang, X., et al., 2011. Synaptic dysfunction and abnormal behaviors in mice lacking major isoforms of Shank3. Hum. Mol. Genet. 20, 3093-3108.

Welch, J.M., et al., 2007. Cortico-striatal synaptic defects and OCD-like behaviours in Sapap3-mutant mice. Nature 448, 894-900.

Williams, D.L., et al., 2005. Verbal and spatial working memory in autism. J. Autism Dev. Disord. 35, 747-756.

Yang, M., et al., 2011. Automated three-chambered social approach task for mice. Curr. Protoc. Neurosci. (Chapter 8, Unit 8 26). 